\title{
Glycine N-methyltransferase deficiency in female mice impairs insulin signaling and promotes gluconeogenesis by modulating the PI3K/Akt pathway in the liver
}

Yi-Jen Liao' ${ }^{1}$, Tzong-Shyuan Lee², Yuh-Ching Twu ${ }^{3}$, Shih-Ming Hsu ${ }^{4}$, Ching-Ping Yang ${ }^{3}$, Chung-Kwe Wang ${ }^{5}$, Yu-Chih Liang ${ }^{1}$ and Yi-Ming Arthur Chen ${ }^{6 *}$

\begin{abstract}
Background: Glycine N-methyltransferase (GNMT) is abundantly expressed in the normal liver but is downregulated in liver cancer tissues. GNMT knockout (Gnmt-/-) mice can spontaneously develop chronic hepatitis, fatty liver, and liver cancer. We previously demonstrated that hepatic GNMT is decreased in high-fat-diet-induced type 2 diabetes mellitus, but its contribution to metabolic syndrome is unclear. Here we show that GNMT modulates key aspects of metabolic syndrome in mice.

Methods: Eleven-week-old Gnmt-/- and wild-type (WT) mice with a C57BL/6 genetic background were used in this study. The metabolic defects of GNMT deficiency were measured by glucose and insulin tolerance tests, lipid homeostasis, gluconeogenesis, and insulin signaling.

Results: Gnmt-/- mice, especially females, exhibited glucose intolerance and insulin resistance. However, their body fat and lean mass, food and water intakes, and energy expenditure did not differ from those of WT mice. In addition, glucose-stimulated insulin secretion and insulin-stimulated glucagon secretion were normal in the serum and pancreatic islets of Gnmt-/- mice. Importantly, we found that GNMT deficiency increased lipogenesis and triglycerides in the liver. The elevated triglycerides disrupted the ability of insulin to induce Akt and S6 ribosomal protein phosphorylation, and then triggered insulin resistance and gluconeogenesis in female Gnmt-/- mice.
\end{abstract}

Conclusions: Our data indicate that hepatic GNMT regulates lipid and glucose homeostasis, and provide insight into the development of insulin resistance through modulating the PI3K/Akt pathway.

Keywords: Glycine N-methyltransferase, Triglycerides, Insulin signaling, PI3K/Akt pathway, Liver

\section{Background}

Metabolic syndrome is a constellation of interrelated disorders that include type 2 diabetes mellitus (T2DM), insulin resistance, dyslipidemia, fatty liver, and atherosclerosis [1]. The pathogenesis of metabolic syndrome is multifactorial, but lipids, glucose, and inflammation manifested as insulin resistance appear to be crucial features [2]. T2DM has reached epidemic proportions worldwide [3]. The rapid increase in the prevalence of

\footnotetext{
* Correspondence: arthur@kmu.edu.tw

${ }^{6}$ Department of Microbiology, College of Medicine, Kaohsiung Medical University, Kaohsiung, Taiwan

Full list of author information is available at the end of the article
}

T2DM in recent decades is due to the interaction of genetic susceptibility and environmental factors such as inappropriate diet and sedentary lifestyles [4-6]. Insulin resistance is correlated with dyslipidemia and nonalcoholic fatty liver disease [7], which is the most common type of liver disease worldwide.

Glycine N-methyltransferase (GNMT) catalyzes the synthesis of sarcosine from glycine using S-adenosylmethionine (SAM) as the methyl donor, and plays an important role in the regulation of the hepatic SAM pool [8]. GNMT also functions as a folate-binding protein and cytosolic receptor for clearing environmental carcinogens by regulating hepatic detoxification pathways $[9,10]$. There is increasing 
evidence that GNMT plays a crucial role in the pathophysiological features of liver diseases, including chronic hepatitis, glycogen storage, hypercholesterolemia, fatty nodules, and liver cancer [11-15]. In addition, a lack of SAM (in a methyl-deficient diet) also causes liver cancer and steatohepatitis in rodents $[16,17]$. A particularly important finding in both our own studies and those of others is that hepatic GNMT is down-regulated in dietary models (e.g., methionine/choline-deficient, high-cholesterol, and high-fat diets) of induced T2DM but not in genetic model (e.g., ob/ob mice) $[18,19]$. Hepatic GNMT is reported elevated in streptozotocin-treated rats [20], a missense mutation $(f a t t y, f a)$ in the leptin receptor gene (ZFD) rats [21], and retinoic acid/dexamethasone-treated rats [22], suggesting that the regulatory mechanisms of GNMT in the liver differ between type 1 diabetes and T2DM, and between dietary and genetic models. Since GNMT is also found in pancreatic tissue [23], whether GNMT is involved in the regulation of insulin signaling and T2DM is largely unknown.

In this study we investigated the role of hepatic GNMT in insulin signaling and the underlying molecular mechanisms. Genetic deletion of GNMT impaired glucose tolerance and insulin sensitivity via the accumulation of triglycerides and deregulation of insulinstimulated Akt activation and gluconeogenesis in the liver.

\section{Methods}

\section{Animals and diet}

Eleven-week-old wild-type (WT) and GNMT knockout (Gnmt-/-) mice [11, 12] with a C57BL/6 genetic background were used in this study. All mice were maintained on standard chow (5001, LabDiet, St Louis, MO, USA) and housed in a 12-/12-h light/dark cycle. The experimental protocols were approved by the Institutional Animal Care and Use Committee of Taipei Medical University.

\section{Glucose and insulin tolerance tests}

For glucose-tolerance tests, the mice $(n=8-10$ per group) were fasted overnight for $16 \mathrm{~h}$. After measuring the fasted blood glucose level, each mouse received intraperitoneal (i.p.) injection of $20 \%$ glucose at $2 \mathrm{~g} / \mathrm{kg}$ body weight (Delta Select, Dreieich, Germany). Blood glucose levels were then measured after $15,30,60,90$, and $120 \mathrm{~min}$. For insulin-tolerance tests, the blood glucose levels were measured after mice had fasted for $3 \mathrm{~h}$. Each mouse received an i.p. insulin injection at $0.75 \mathrm{U} /$ kg body weight (Actrapid, Novo Nordisk, Bagsvaerd, Denmark), and the blood glucose levels were then measured after 15, 30, 45, and $60 \mathrm{~min}$.

\section{Physiological metabolic analyses}

The metabolic rate, food and water intakes and body fat and lean mass of WT and Gnmt-/- mice ( $n=3$ or 4 per group) were analyzed using the LabMaster Calorimetry Module (TSE Systems, Bad Homburg, Germany), Tecniplast device, and Skyscan 1076 device, respectively.

\section{Glucagon and insulin secretion}

All animals ( $n=4-7$ per group) received an i.p. injection with insulin (100 IU/ml, Actrapid, Novo Nordisk, Bagsvaerd, Denmark) at $0.75 \mathrm{U} / \mathrm{kg}$ body weight or $20 \%$ glucose at $2 \mathrm{~g} / \mathrm{kg}$ body weight. After $20 \mathrm{~min}$, the pancreatic and blood samples were collected for immunohistochemistry (IHC) staining and ELISA analyses.

\section{IHC and ELISA}

Paraffin-embedded pancreatic sections were incubated with the antibodies against glucagon (1:200 dilution, Lot No. \#8233) and insulin (1:100 dilution, Lot No. \#4590) (Cell Signaling, Beverly, MA, USA) and detected using the Universal LSAB $^{\mathrm{TM} 2}$ kit (DakoCytomation Carpinteria, CA, USA) according to the manufacturer's instructions. The serum insulin levels were measured using mouse insulin ELISA kit (Cat. \#EZRMI-13 K, Millipore, Billerica, MA) in accordance with the manufacturer's instructions. The whole blood samples were directly drawn into a centrifuge tube without anti-coagulant. Let blood clot at room temperature for $30 \mathrm{~min}$. Centrifuge the clotted blood at 2,000 to $3,000 \times \mathrm{g}$ for $15 \mathrm{~min}$ at $4{ }^{\circ} \mathrm{C}$ and the serum samples were subjected to insulin quantification.

\section{Western blotting}

Liver tissues were lysed using a lysis buffer supplemented with protease and phosphatase inhibitors [24]. Proteins $(50 \mu \mathrm{g})$ were separated using an $8-12 \%$ SDS gel. The following antibodies (1:1000 dilution) used in this study were purchased from Cell Signaling: phosphoAkt (Ser-473, Lot No. \#4060), total-Akt (Lot No. \#4691), phospho-S6 ribosomal protein (Ser240/244, Lot No. \#4858; and Ser235/236, Lot No. \#5364) and total-S6 ribosomal protein (Lot No.\#2317), and phospho-insulin receptor $\beta$ (IR- $\beta$, Tyr1150/1151, Lot No. \#3024). TotalIR- $\beta$ (Lot No. sc-711) was purchased from Santa Cruz Biotechnology. Immunoblotting signals were normalized to those for $\alpha$-tubulin (1:5000 dilution, Lot No. T9026, Sigma-Aldrich), and quantified by densitometric scanning.

\section{Gene expression analysis}

Total RNA was isolated from mouse liver using TRIzol Reagent (Ambion, Carlsbad, CA, USA), according to the manufacturer's protocol. Complementary DNA was produced from RNA ( $2 \mu \mathrm{g})$ using a SuperScript II RNase HReverse Transcriptase Kit (Invitrogen, Carlsbad, CA, 
USA). The real-time PCR reactions using SYBR Green (Applied Biosystems, Foster City, CA, USA) were assayed using an Applied Biosystems Prism 7700 sequence detection system. The specific primer sequences are listed in Additional file 1: Table S1. Predicted cycle threshold (CT) values were exported into Microsoft Excel worksheets for analysis. Comparative CT methods were used to determine the differences in gene expression relative to GAPDH. Relative amounts of mRNA level in Gnmt-/- mice were determined by calculating the ratio relative to the WT mice separately for each sex.

\section{Statistical analysis}

Statistical analyses were performed using SPSS version 13 (SPSS, Chicago, IL, USA). Student's $t$-test was used to compare groups. A probability value of $p<0.05$ was considered to indicate statistical significance.

\section{Results}

GNMT deficiency impaired glucose tolerance, especially in female mice

To investigate whether GNMT deficiency contributes to the metabolic phenotype arising from glucose intolerance and insulin resistance, we applied a glucose tolerance test and an insulin tolerance test to a Gnmt-/- mouse model. As shown in Fig. 1a, we detected an impaired glucose tolerance in female Gnmt-/- mice at 30 and 60 min after glucose administration. On the other hand, GNMT deficiency also impaired insulin sensitivity in female mice
(Fig. 1b). In contrast, the findings of both glucose tolerance and insulin sensitivity tests did not differ between male Gnmt-/- mice and their WT littermate control mice (Fig. 1). These data demonstrate that compared to WT mice, female Gnmt-/- mice was more glucose intolerant and insulin resistant than male Gnmt-/- mice.

\section{GNMT deficiency did not impair the metabolic effects}

Considering the effects of GNMT on controlling energy homeostasis, we first analyzed the body composition of Gnmt-/- mice. The body fat and body lean mass did not differ between Gnmt-/- and WT mice (Fig. 2a). Alterations in the basal metabolic rate were compared by carrying out a metabolic characterization in Gnmt-/mice. The daily food and water intakes did not differ between Gnmt-/- and WT mice of the same sex (Fig. 2b). The daily increase in body weight was the same in Gnmt -/- and WT mice (Fig. 2c). Calorimetric experiments were conducted during the fed state. The respiratory quotient did not differ between Gnmt-/- and WT mice (Fig. 2d).

\section{GNMT deficiency did not impair the secretion function of pancreatic islets}

To obtain a better understanding of the impact of the GNMT gene knockout on pancreatic function, we first measured the insulin levels in WT and Gnmt-/- mice. The fasting serum insulin levels did not differ between Gnmt-/- mice and their WT

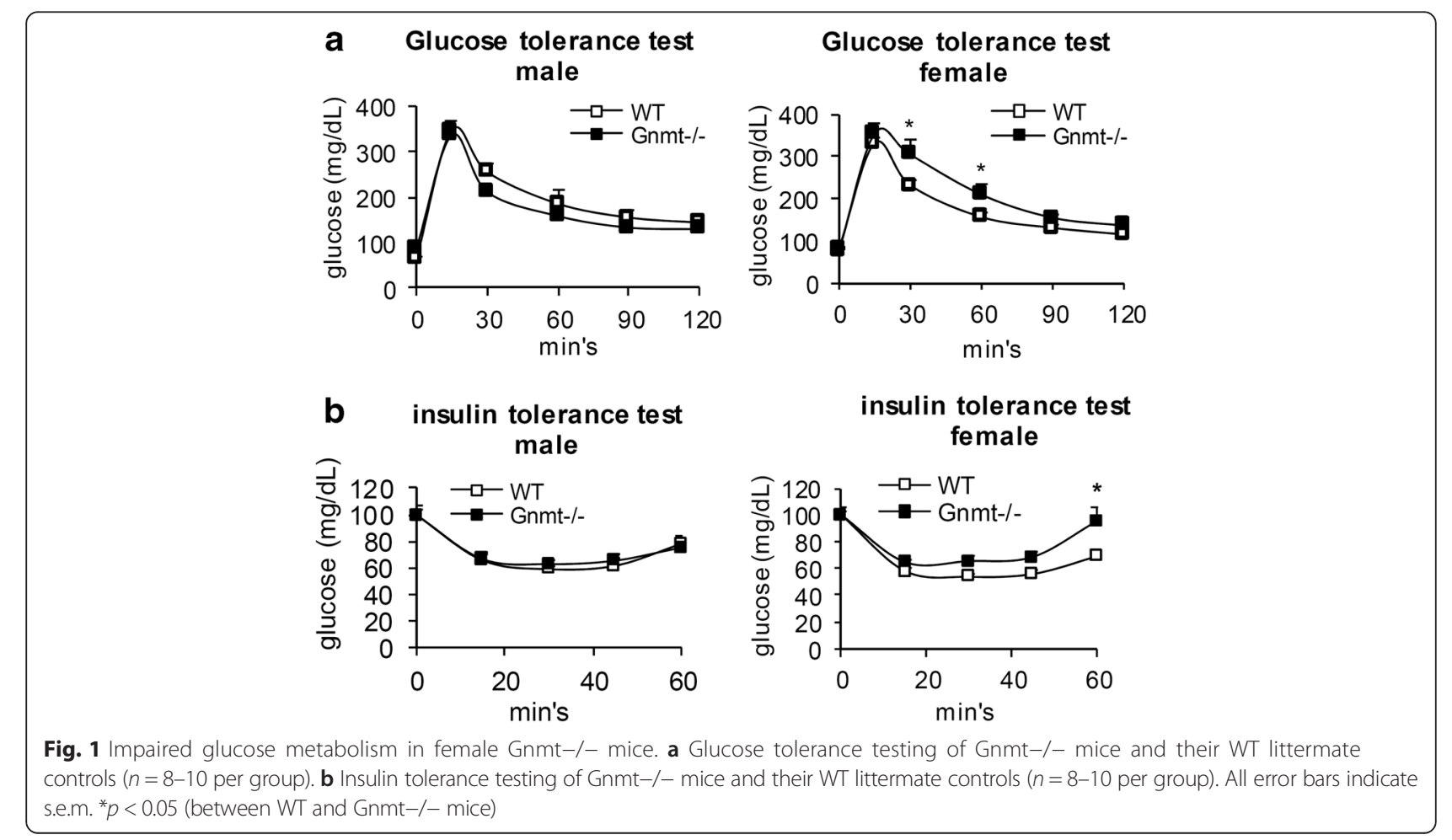



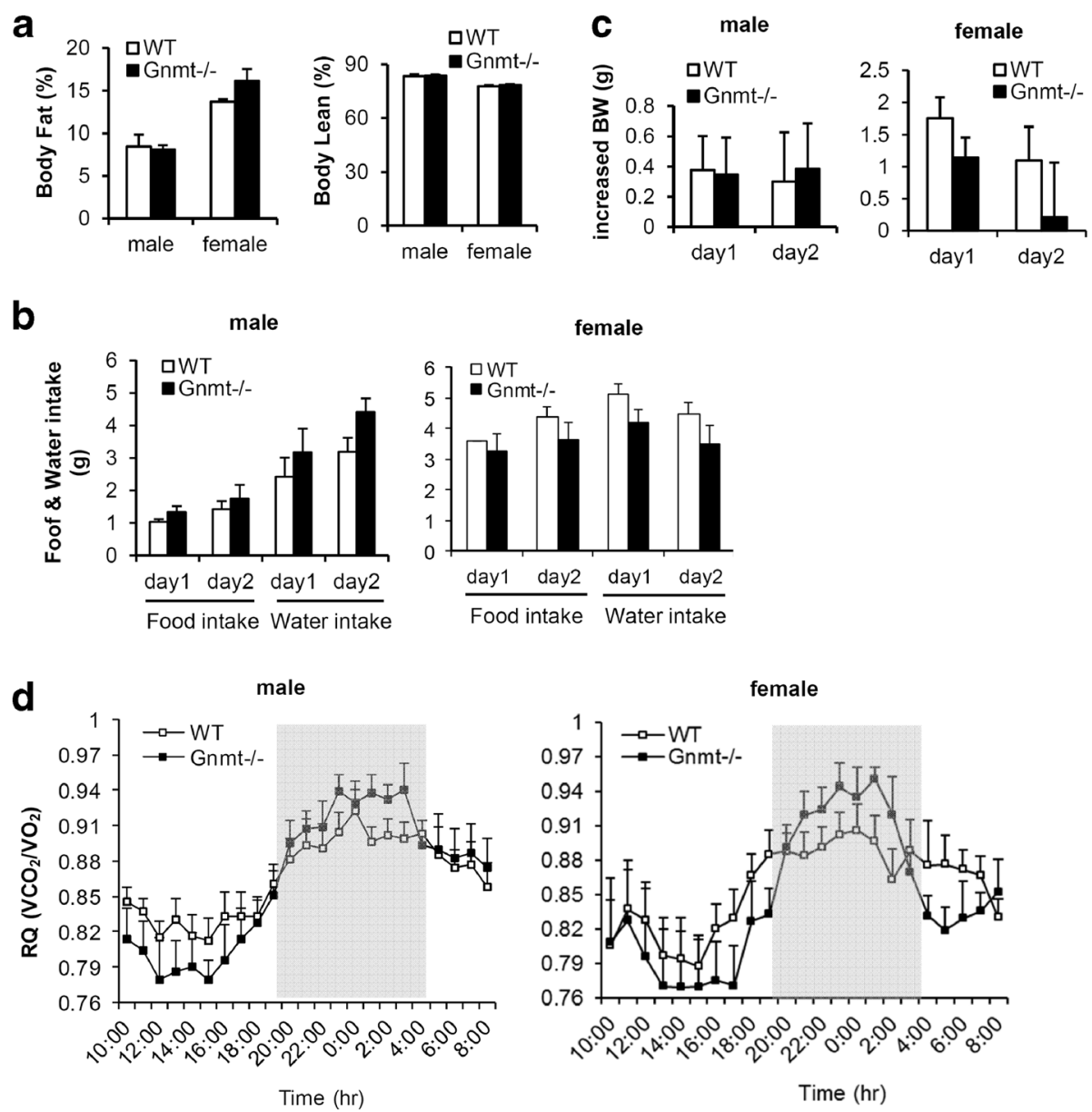

Fig. 2 GNMT deficiency did not impair the metabolic effects. a Body fat and lean mass, b food and water intakes, and $\mathbf{c}$ increased body weight (BW) of WT and Gnmt-/- mice over 2 days ( $n=3$ or 4 per group). $\mathbf{d}$ Respiratory quotient (RQ) in WT and Gnmt-/- mice over $24 \mathrm{~h}$ ( $n=3$ or 4 per group). All error bars indicate s.e.m

littermate controls for either sex (Fig. 3a). We then analyzed glucose-stimulated insulin secretion and insulin-stimulated glucagon secretion in pancreatic islets. As shown in Fig. $2 \mathrm{~b}$ and c, glucose treatment induced normal insulin secretion. In addition, insulin-stimulated glucagon secretion in $\alpha$-cell islets exhibited no alterations between Gnmt-/- and WT mice of either sex (Fig. 3c). These data suggest that impaired glucose metabolism in Gnmt-/- mice arises primarily from hepatic insulin resistance rather than impaired insulin secretion due to $\beta$-cell dysfunction.
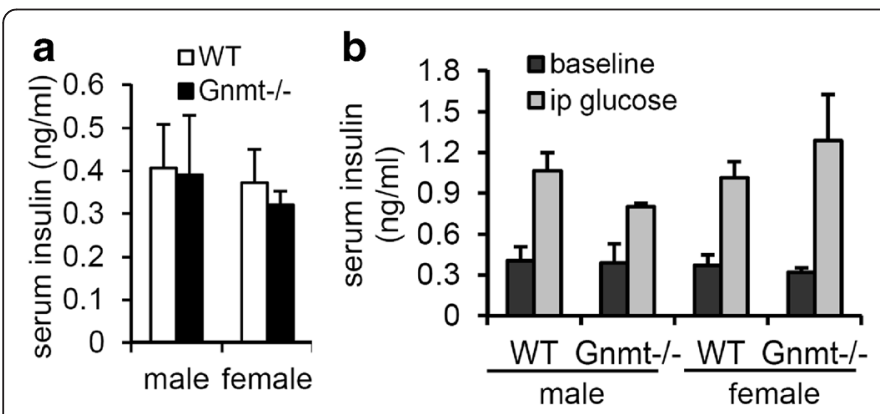

\section{C}

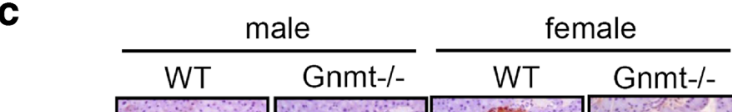

insulin

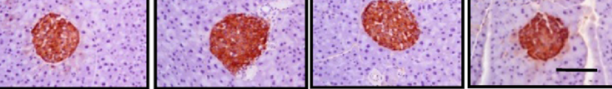

glucagon

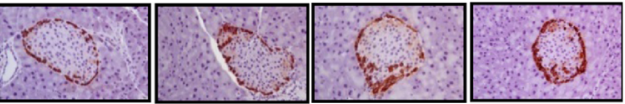

Fig. 3 GNMT deficiency did not impair the secretion function of pancreatic islets. a Serum insulin levels in overnight-fasted WT and Gnmt-/mice ( $n=5$ per group). b Serum insulin levels after glucose injection in WT and Gnmt-/- mice mice ( $n=4-7$ per group). $\mathbf{c}$ Insulin and glucagon staining of pancreatic islets in WT and Gnmt-/- mice. Scale bars indicate $100 \mu \mathrm{m}$. All error bars indicate s.e.m 
Increased lipogenesis and accumulated triglycerides in the liver of Gnmt-/- mice

The accumulation of hepatic triglycerides is strongly associated with insulin resistance $[7,25]$. Since we found impaired systemic insulin sensitivity in female Gnmt-/mice (Fig. 1), we subsequently evaluated the hepatic steatosis and de novo lipogenesis in these mice. Steatosis was quantified by measuring the total hepatic triglycerides level, which revealed a two-fold increase in Gnmt -/- mice relative to WT mice (Fig. 4a). In addition, the serum levels of triglycerides and very low density lipoprotein (VLDL) were both decreased in Gnmt-/- mice (Fig. 4b). Increased de novo lipogenesis and decreased VLDL secretion are crucial for the development of hepatic steatosis [25], and so we measured the expression of transcription factors that promote hepatic lipogenesis as well as genes that encode enzymes that contribute to lipogenesis. The levels of peroxisome proliferatoractivated receptor- $\gamma$ (PPAR $\gamma$ ) and sterol-regulatory element-binding protein 1c (Srebp1c), which are two key regulators of lipogenetic genes in response to insulin, did not differ between WT and Gnmt-/- mice (Fig. 4c). On the other hand, fatty acid synthase (Fasn), microsomal triglyceride transfer protein (Mttp), long-chain fatty-acid-CoA ligase-4 (Ascl4), stearoyl-CoA desaturase 1 (Scd1), and Acetyl-CoA carboxylase (Acc), which are genes involved in de novo lipogenesis, were significantly zelevated in female Gnmt-/- mice relative to WT mice (Fig. 4c). Importantly, those genes involved in de novo lipogenesis were also significantly elevated in female Gnmt-/- mice relative to male Gnmt-/- mice (Fig. 4c). These data imply that the hepatic insulin resistance observed in female Gnmt-/- mice may due to increased de novo lipogenesis.

\section{Dysregulation of insulin-stimulated Akt activation in the liver of female Gnmt-/- mice}

To further investigate the molecular basis of insulin resistance in Gnmt-/- mice, we analyzed insulin-stimulated signaling in the liver. The i.p. injection of insulin induced the phosphorylation of the IR- $\beta$, S6 ribosomal protein and Akt in WT and Gnmt-/- mice (Fig. 5). Importantly, the activation level of S6 ribosomal protein and Akt was much lower in female Gnmt-/- mice than in WT mice (Fig. 5b). The phosphorylation of IR- $\beta$ was enhanced in insulinstimulated WT and Gnmt-/- mice, however, female Gnmt-/- mice expressed more significance phosphorylation of IR- $\beta$ (Fig. 5b). While, the male Gnmt-/- mice showed less phosphorylation of IR- $\beta$ (Fig. 5a).

\section{GNMT knockdown is associated with increased expression} of liver gluconeogenesis genes in female mice

To assess the mechanism underlying the impairment of whole body glucose homeostasis in female Gnmt-/-

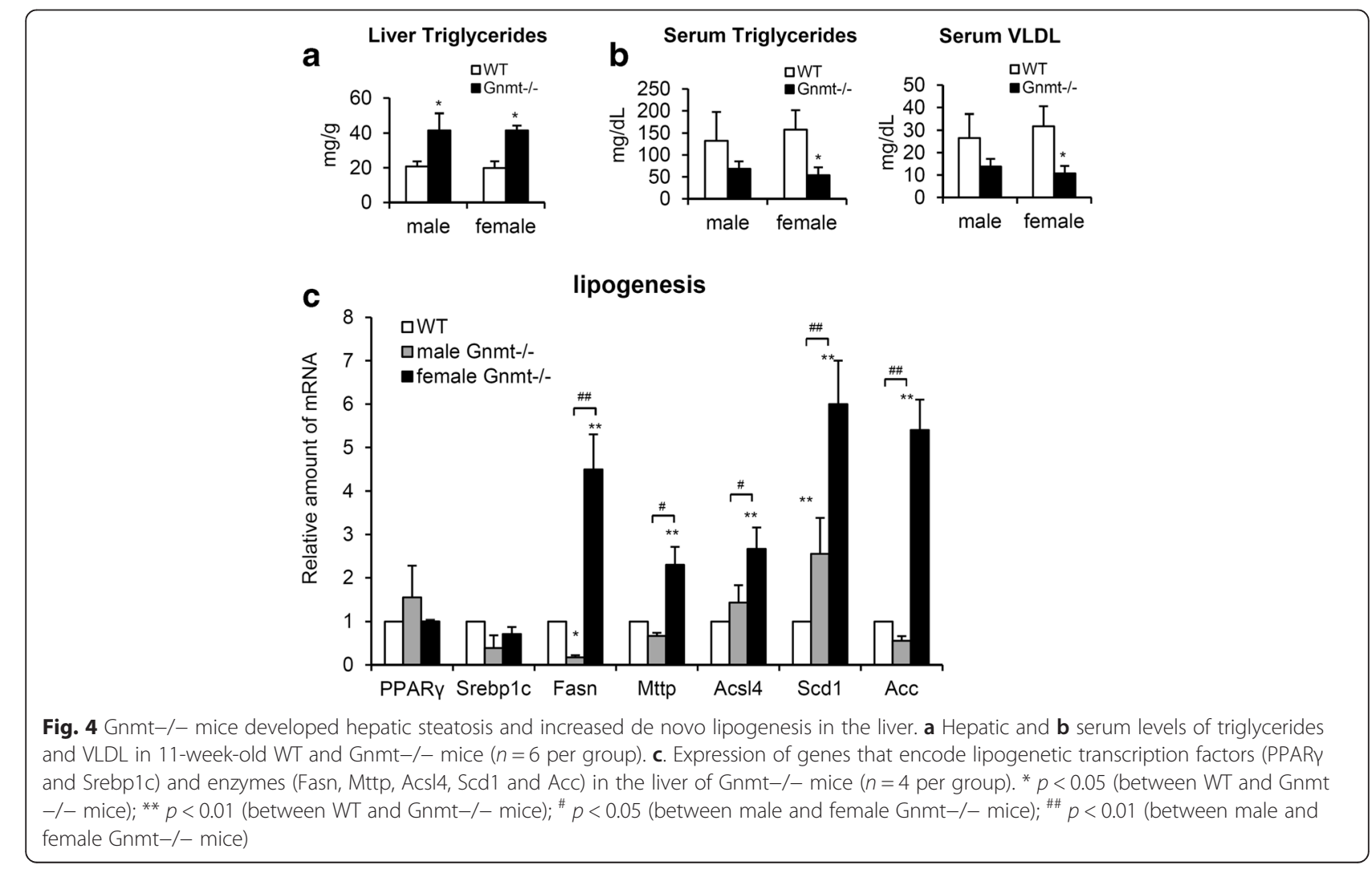



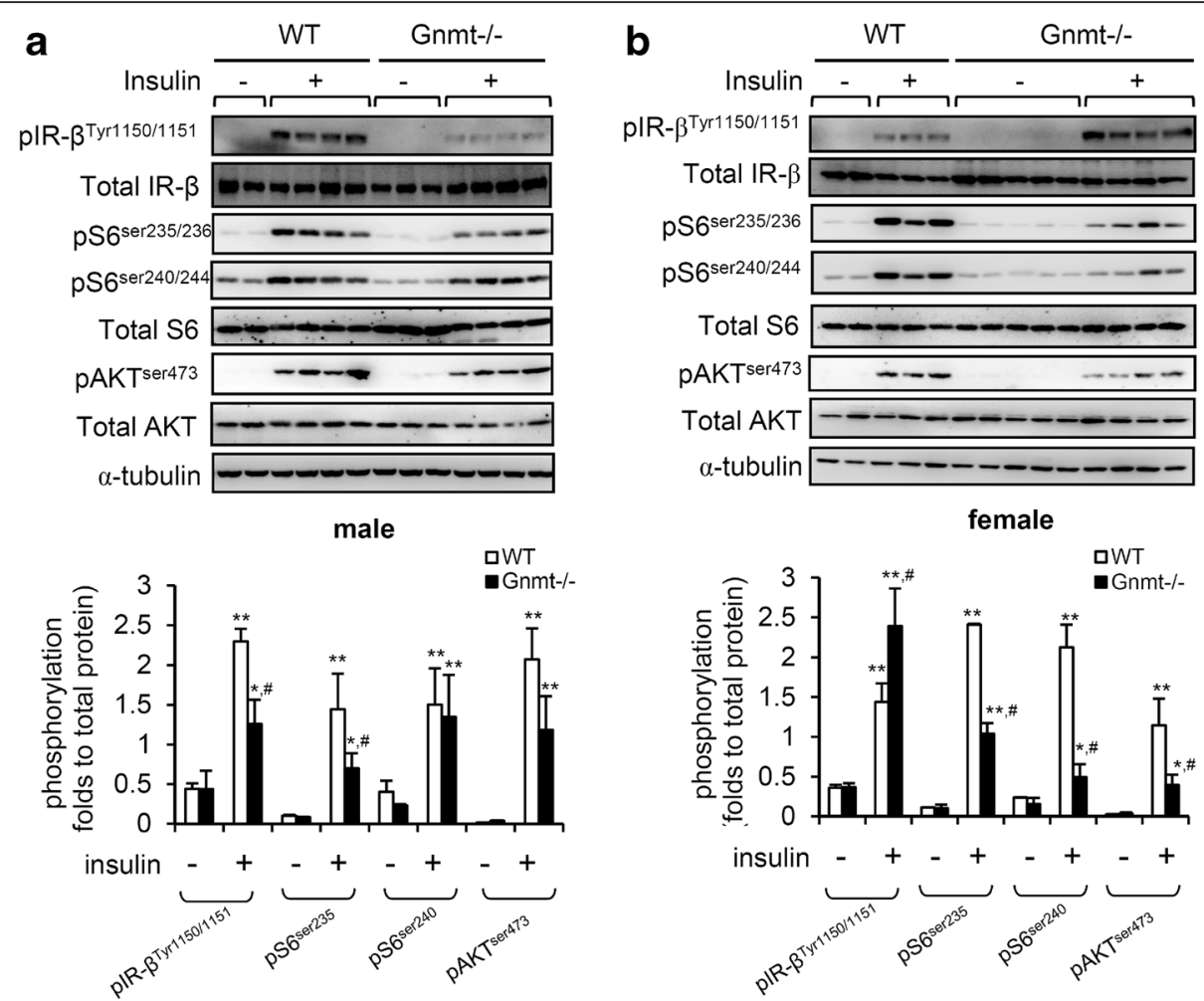

Fig. 5 GNMT deficiency impaired insulin-stimulated Akt and S6 ribosomal protein activation in the liver. Representative Western blot analysis and quantification of expression and insulin-stimulated phosphorylation of Akt, S6 ribosomal protein, and IR in the liver of male $\mathbf{a}$ and female $\mathbf{b}$ WT and Gnmt-/- mice ( $n=4-5$ per group). $a-T u b u l i n$ was used as a loading control. The levels of immunoreactive phosphorylated proteins were normalized to the total expression of the respective protein. All error bars indicate s.e.m. ${ }^{* *} p<0.01$ (between baseline and insulin treatment); ${ }^{*} p<0.05$ (between baseline and insulin treatment); ${ }^{*} p<0.05$ (between insulin-treated WT and Gnmt-/- mice)

mice (Fig. 1), we analyzed the expression of genes involved in gluconeogenesis. Consistent with the physiological data obtained in the glucose tolerance test (Fig. 1) and signaling data (Fig. 5b), female Gnmt-/mice displayed significant elevations of gluconeogenic markers (Pepck, Foxo1, and G6pase) in the liver relative to male Gnmt-/- mice (Fig. 6).

\section{Discussion and conclusion}

GNMT is abundantly expressed in normal livers, but is down-regulated in high fat diet-induced T2DM and liver cancer tissues $[13,19]$. GNMT deficiency in mice results in chronic hepatitis, fatty liver, and liver cancer spontaneously $[11,12,15]$. However, the molecular mechanisms underlying GNMT-deficiency-induced hepatic triglycerides accumulation and insulin signaling remain unclear. Excess SAM has been found to increase the turnover of hepatic triglycerides stored for secretion in VLDL in Gnmt-/- mice [26]. The findings of the current study support the novel concept of GNMT deficiency enhancing lipogenesis and triglycerides accumulation, subsequently triggering insulin resistance and gluconeogenesis by modulating the PI3K/ Akt pathway in the liver (Fig. 7).

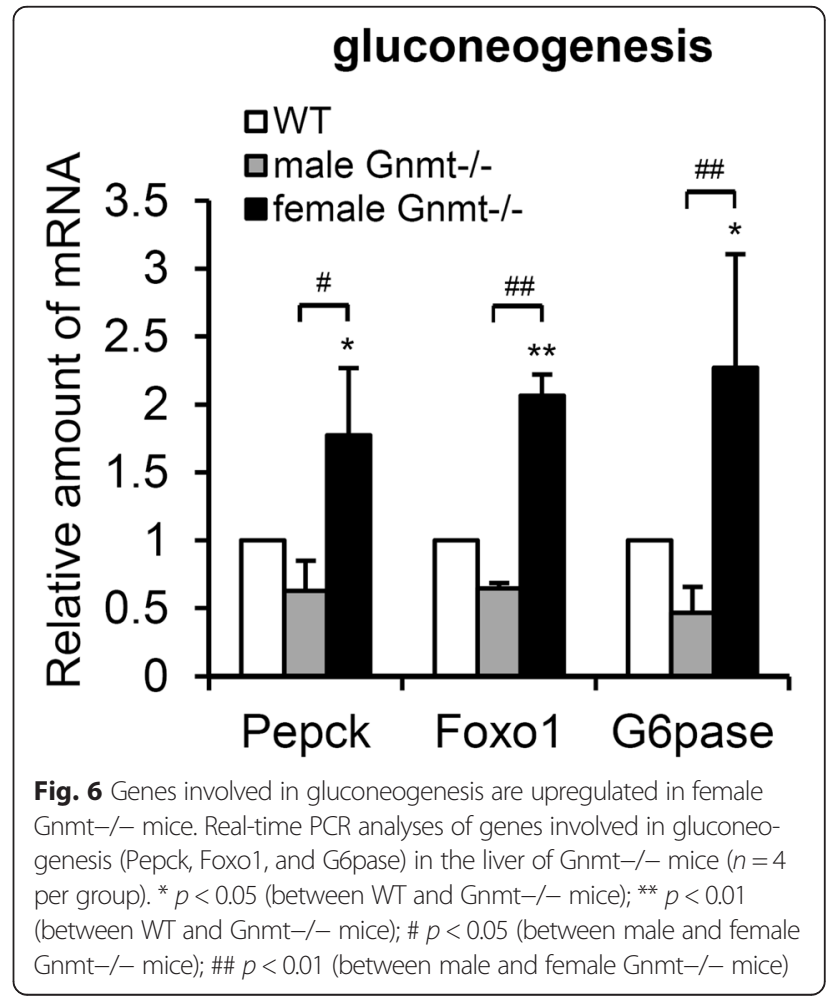




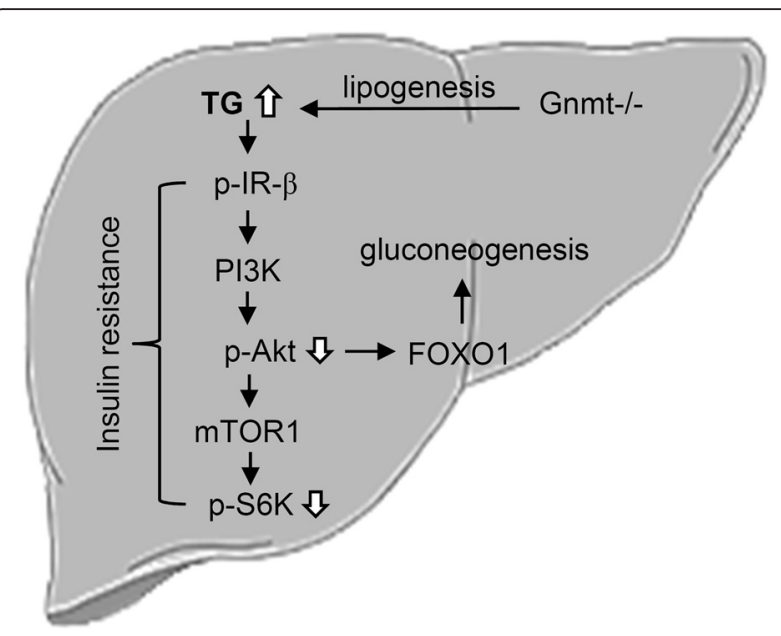

Fig. 7 Illustration of proposed mechanism underlying GNMT deficiency induced triglycerides accumulation and insulin resistance in the liver. GNMT deficiency may enhance lipogenesis to cause hepatic triglycerides accumulation, thus impairing insulin signaling and gluconeogenesis, especially in female Gnmt-/- mice

The liver plays a critical role in the systemic response to insulin, controlling the metabolism of both glucose and lipids [27, 28]. Hepatic lipogenesis includes the de novo synthesis of fatty acids from acetyl-CoA and malonyl-CoA and further processing into triglycerides. The produced triglycerides are then either stored in lipid droplets or packed into VLDL and exported into the blood [28]. In our study we found that the increased de novo lipogenesis and inhibition of hepatic triglycerides exported via VLDL contribute to lipid accumulation in the liver of Gnmt -/- mice (Fig. 4). Triglycerides, which are the main lipids stored in the liver of nonalcoholic fatty liver disease patients, are associated with the development of steatohepatitis and insulin resistance [7, 25, 29]. Indeed, hepatic inflammatory infiltration [19] as well as inflammatory genes (IL-6, TNF- $\alpha$ and IL1- $\beta$ ) were increased in Gnmt-/- mice (data not shown). The impairment of PI3K/Akt activation is involved in the triglycerides accumulation induced hepatic insulin resistance [7, 30]. Our study has revealed impaired insulin-stimulated Akt phosphorylation in Gnmt-/mice (Fig. 5).

Our previous studies suggested that the rate of tumorigenesis in $\mathrm{HCC}$ was significantly higher in female Gnmt-/- mice [10-12, 31]. Moreover, the retinoids that induce the activation of GNMT are sexspecific [32]. In the present study we observed that female Gnmt-/- mice express a glucose intolerance phenotype (Fig. 1). The activation of the PI3K/Akt pathway is responsible for glucose metabolism, including glycogen synthesis and gluconeogenesis [33]. We found that the gluconeogenesis genes (Pepck,
Foxo1, and G6pase) were up-regulated only in female Gnmt-/- mice (Fig. 6). Foxo1 stimulates hepatic gluconeogenic gene expression and is directly phosphorylated and inhibited by Akt [34, 35]. Indeed, the level of insulin-stimulated Akt phosphorylation was lower in female Gnmt-/- mice (Fig. 5b). In addition, a twofold increase in hepatic triglycerides was observed in Gnmt-/- mice of either sex, but the lipogenesis and gluconeogenesis were elevated in female Gnmt-/mice. Although the detailed underlying mechanism is unclear, this discrepancy may due to different roles of estrogen and androgen in Gnmt-/- mice.

Previously, we reported that GNMT regulates liver cancer growth in part via interacting with the DEP domain containing mTOR-interacting protein (DEPTOR) and modulating the mTOR signaling pathway [24]. DEPTOR is an mTOR inhibitor that interacts with mTOR directly. Overexpression of DEPTOR activates Akt via the inhibition of a negative feedback loop from S6 to PI3K [36]. The phosphorylation of IR- $\beta$ is able to recruit and phosphorylate IR substrate proteins after ligand binding, leading to activation of the PI3K/Akt pathway $[37,38]$. However, the difference of insulin-stimulated pIR- $\beta$ was observed between male and female Gnmt-/- mice (Fig. 5). Although the detailed underlying mechanism is unclear, this sex discrepancy may due to different feedback controls of hepatic glucose metabolism [12], methylation [11] or hormone [32] in Gnmt-/- mice.

GNMT is expressed predominantly in the liver, with moderate levels in proximal kidney tubules and exocrine tissue of the pancreas [8, 23]. However, GNMT deficiency does not affect the insulin and glucagon secretion of pancreatic islets (Fig. 3), implying that the dysregulation of glucose metabolism in Gnmt-/mice may arise primarily from hepatic insulin resistance rather than impaired insulin secretion due to $\beta$-cell dysfunction. In addition to the liver, skeletal muscle and adipocytes are the main insulinresponsive tissues and are associated with the pathogenesis of T2DM [30]. Although we did not detect GNMT in adipocytes using Western blotting and Q-PCR, weak expressions of GNMT in both mRNA and protein levels were observed in the skeletal soleus muscle (data not shown). Therefore, the role of GNMT in these insulin target organs is yet to be elucidated, especially in skeletal muscle.

In conclusion, our findings indicate that GNMT deficiency exacerbates lipogenesis and the accumulation of triglycerides in the liver, further impairing insulin signaling and gluconeogenesis. GNMT may therefore be a suitable therapeutic target when treating not only dyslipidemia but also broader aspects of metabolic diseases. 


\section{Additional file}

Additional file 1: Table S1. Real-time PCR primers used in the study. (DOC $38 \mathrm{~kb}$ )

\section{Abbreviations}

Acc, acetyl-CoA carboxylase; Ascl4, long-chain fatty-acid-CoA ligase-4; DEPTOR DEP domain containing mTOR-interacting protein; Fasn, fatty acid synthase; GNMT, glycine N-methyltransferase; Gnmt-/-, GNMT knockout; IR- $\beta$, insulin receptor $\beta$; Mttp, microsomal triglyceride transfer protein; PPARY, peroxisome proliferator-activated receptor- $\gamma$; SAM, s-adenosylmethionine; Scd1, stearoyl-CoA desaturase 1; Srebp1c, sterol-regulatory element-binding protein 1c; T2DM, type 2 diabetes mellitus; WT, wild-type.

\section{Acknowledgements}

We thank the Taiwan Mouse Clinic (MOST 103-2325-B-001-015) which is funded by the National Research Program for Biopharmaceuticals (NRPB) at the Ministry of Science and Technology (MOST) of Taiwan for technical support in physiological metabolic experiments. We also thank Yuan-Hsi Wang for support in Q-PCR experiments.

\section{Funding}

This study was partially supported by Ministry of Science and Technology of the Republic of China (MOST104-2632-B-037-001 and MOST103-2320-B-038044) and Kaohsiung Medical University (KMU-TP104E11).

\section{Availability of data and materials}

Not applicable.

\section{Authors' contributions}

Conception and design: YJL, YMC. Analysis and interpretation of data: YJL, TSL, YCT, CPY. Administrative, technical, or material support: SMH, CKW, YCL. Wrote the manuscript: YJL. All authors read and approved the final manuscript.

\section{Competing interests}

The authors have no conflicts of interest.

\section{Consent for publication}

Not applicable.

\section{Ethics approval and consent to participate}

The experimental protocols were approved by the Institutional Animal Care and Use Committee of Taipei Medical University (LAC-2013-0277)

\section{Author details}

${ }^{1}$ School of Medical Laboratory Science and Biotechnology, College of Medical Science and Technology, Taipei Medical University, Taipei, Taiwan. ${ }^{2}$ Department and Institute of Physiology, National Yang-Ming University, Taipei, Taiwan. ${ }^{3}$ Department of Biotechnology and Laboratory Science in Medicine, School of Biomedical Science and Engineering, National Yang-Ming University, Taipei, Taiwan. ${ }^{4}$ Department of Biomedical Imaging and Radiological Sciences, National Yang-Ming University, Taipei, Taiwan. ${ }^{5}$ Department of International Medicine, Taipei City Hospital Ranai Branch, Taipei, Taiwan. ${ }^{6}$ Department of Microbiology, College of Medicine, Kaohsiung Medical University, Kaohsiung, Taiwan.

\section{Received: 1 April 2016 Accepted: 30 July 2016}

Published online: 04 October 2016

\section{References}

1. Gaggini M, Morelli M, Buzzigoli E, DeFronzo RA, Bugianesi E, Gastaldelli A. Non-alcoholic fatty liver disease (NAFLD) and its connection with insulin resistance, dyslipidemia, atherosclerosis and coronary heart disease. Nutrients. 2013;5:1544-60.

2. Medina-Santillan R, Lopez-Velazquez JA, Chavez-Tapia N, Torres-Villalobos G, Uribe M, Mendez-Sanchez N. Hepatic manifestations of metabolic syndrome. Diabetes/metabolism research and reviews. 2013. doi: 10.1002/ dmrr.2410.
3. Shaw JE, Sicree RA, Zimmet PZ. Global estimates of the prevalence of diabetes for 2010 and 2030. Diabetes Res Clin Pract. 2013:87:4-14.

4. Diamant M, Blaak EE, de Vos WM. Do nutrient-gut-microbiota interactions play a role in human obesity, insulin resistance and type 2 diabetes? Obes Rev. 2011;12:272-81.

5. Danaei G, Finucane MM, Lu Y, Singh GM, Cowan MJ, Paciorek CJ, et al. National, regional, and global trends in fasting plasma glucose and diabetes prevalence since 1980: systematic analysis of health examination surveys and epidemiological studies with 370 country-years and 2.7 million participants. Lancet. 2013;378:31-40.

6. Whiting DR, Guariguata L, Weil C, Shaw J. IDF diabetes atlas: global estimates of the prevalence of diabetes for 2011 and 2030. Diabetes Res Clin Pract. 2013:94:311-21.

7. Birkenfeld AL, Shulman GI. Nonalcoholic fatty liver disease, hepatic insulin resistance, and type 2 diabetes. Hepatology. 2014:59:713-23.

8. Kerr SJ. Competing methyltransferase systems. J Biol Chem. 1972;247:4248-52.

9. Cook RJ, Wagner C. Glycine N-methyltransferase is a folate binding protein of rat liver cytosol. Proc Natl Acad Sci U S A. 1984;81:3631-4.

10. Liao YJ, Chen KH, Huang SF, Chen TL, Wang CK, Chien $\mathrm{CH}$, et al. Deficiency of glycine $\mathrm{N}$-methyltransferase results in deterioration of cellular defense to stress in mouse liver. Proteomics Clin Appl. 2010:4:394-406.

11. Liao YJ, Liu SP, Lee CM, Yen CH, Chuang PC, Chen CY, et al. Characterization of a glycine $\mathrm{N}$-methyltransferase gene knockout mouse model for hepatocellular carcinoma: Implications of the gender disparity in liver cancer susceptibility. Int J Cancer. 2009;124:816-26.

12. Liu SP, Li YS, Chen YJ, Chiang EP, Li AF, Lee YH, et al. Glycine Nmethyltransferase-/- mice develop chronic hepatitis and glycogen storage disease in the liver. Hepatology. 2007:46:1413-25.

13. Chen YM, Shiu JY, Tzeng SJ, Shih LS, Chen YJ, Lui WY, et al. Characterization of glycine-N-methyltransferase-gene expression in human hepatocellular carcinoma. Int J Cancer. 1998;75:787-93.

14. Liu HH, Chen KH, Shih YP, Lui WY, Wong FH, Chen YM. Characterization of reduced expression of glycine $\mathrm{N}$-methyltransferase in cancerous hepatic tissues using two newly developed monoclonal antibodies. J Biomed Sci. 2003;10:87-97.

15. Martinez-Chantar ML, Vazquez-Chantada M, Ariz U, Martinez N, Varela M, Luka Z, et al. Loss of the glycine N-methyltransferase gene leads to steatosis and hepatocellular carcinoma in mice. Hepatology. 2008;47:1191-9.

16. Pogribny IP, James SJ, Beland FA. Molecular alterations in hepatocarcinogenesis induced by dietary methyl deficiency. Mol Nutr Food Res. 2012;56:116-25.

17. Mato JM, Lu SC. The hepatocarcinogenic effect of methionine and choline deficient diets: an adaptation to the Warburg effect? Alcohol Clin Exp Res. 2011:35:811-4.

18. Pacana T, Cazanave S, Verdianelli A, Patel V, Min HK, Mirshahi F, et al. Dysregulated hepatic methionine metabolism drives homocysteine elevation in diet-induced nonalcoholic fatty liver disease. PloS One. 2015;10:e0136822.

19. Liao YJ, Chen TL, Lee TS, Wang HA, Wang CK, Liao LY, et al. Glycine Nmethyltransferase deficiency affects Niemann-Pick type C2 protein stability and regulates hepatic cholesterol homeostasis. Mol Med. 2012;18:412-22.

20. Nieman KM, Schalinske KL. Insulin administration abrogates perturbation of methyl group and homocysteine metabolism in streptozotocin-treated type 1 diabetic rats. Am J Physiol Endocrinol Metab. 2011;301:E560-565.

21. Williams KT, Schalinske KL. Tissue-specific alterations of methyl group metabolism with DNA hypermethylation in the Zucker (type 2) diabetic fatty rat. Diabetes Metab Res Rev. 2012;28:123-31.

22. Rowling MJ, Schalinske KL. Retinoic acid and glucocorticoid treatment induce hepatic glycine $\mathrm{N}$-methyltransferase and lower plasma homocysteine concentrations in rats and rat hepatoma cells. J Nutr. 2003; 133:3392-8.

23. Yeo EJ, Wagner $\mathrm{C}$. Tissue distribution of glycine $\mathrm{N}$-methyltransferase, a major folate-binding protein of liver. Proc Natl Acad Sci U S A. 1994;91:210-4.

24. Yen CH, Lu YC, Li CH, Lee CM, Chen CY, Cheng MY, et al. Functional characterization of glycine $\mathrm{N}$-methyltransferase and its interactive protein DEPDC6/DEPTOR in hepatocellular carcinoma. Mol Med. 2012;18:286-96.

25. Tilg $H$, Moschen AR. Insulin resistance, inflammation, and non-alcoholic fatty liver disease. Trends Endocrinol Metab. 2008;19:371-9.

26. Martinez-Una M, Varela-Rey M, Mestre D, Fernandez-Ares L, Fresnedo O, Fernandez-Ramos D, et al. S-Adenosylmethionine increases circulating very-low density lipoprotein clearance in non-alcoholic fatty liver disease. J Hepatol. 2015;62:673-81. 
27. Maxfield FR, Tabas I. Role of cholesterol and lipid organization in disease. Nature. 2005;438:612-21.

28. Bechmann LP, Hannivoort RA, Gerken G, Hotamisligil GS, Trauner M, Canbay A. The interaction of hepatic lipid and glucose metabolism in liver diseases. J Hepatol. 2012;56:952-64.

29. Asrih M, Jornayvaz FR. Metabolic syndrome and nonalcoholic fatty liver disease: Is insulin resistance the link? Mol Cell Endocrinol. 2015;418:55-65.

30. Samuel VT, Shulman Gl. Mechanisms for insulin resistance: common threads and missing links. Cell. 2012;148:852-71.

31. Liu SP, Li YS, Lee CM, Yen CH, Liao YJ, Huang SF, et al. Higher susceptibility to aflatoxin $\mathrm{B}(1)$-related hepatocellular carcinoma in glycine $\mathrm{N}$-methyltransferase knockout mice. Int J Cancer. 2010;128:511-23.

32. McMullen MH, Rowling MJ, Ozias MK, Schalinske KL. Activation and induction of glycine $\mathrm{N}$-methyltransferase by retinoids are tissue- and gender-specific. Archives of biochemistry and biophysics. 2002:401:73-80.

33. Perry RJ, Samuel VT, Petersen KF, Shulman GI. The role of hepatic lipids in hepatic insulin resistance and type 2 diabetes. Nature. 2014;510:84-91.

34. Gross DN, Wan M, Birnbaum MJ. The role of FOXO in the regulation of metabolism. Curr Diab Rep. 2009;9:208-14.

35. Lu M, Wan M, Leavens KF, Chu Q, Monks BR, Fernandez S, et al. Insulin regulates liver metabolism in vivo in the absence of hepatic Akt and Foxo1. Nat Med. 2012;18:388-95.

36. Peterson TR, Laplante M, Thoreen CC, Sancak Y, Kang SA, Kuehl WM, et al. DEPTOR is an $\mathrm{mTOR}$ inhibitor frequently overexpressed in multiple myeloma cells and required for their survival. Cell. 2009;137:873-86.

37. Biddinger SB, Kahn CR. From mice to men: insights into the insulin resistance syndromes. Annu Rev Physiol. 2006;68:123-58.

38. Sun XJ, Rothenberg P, Kahn CR, Backer JM, Araki E, Wilden PA, et al. Structure of the insulin receptor substrate IRS-1 defines a unique signal transduction protein. Nature. 1991;352:73-7.

\section{Submit your next manuscript to BioMed Central and we will help you at every step:}

- We accept pre-submission inquiries

- Our selector tool helps you to find the most relevant journal

- We provide round the clock customer support

- Convenient online submission

- Thorough peer review

- Inclusion in PubMed and all major indexing services

- Maximum visibility for your research

Submit your manuscript at www.biomedcentral.com/submit

CBiomed Central 